\title{
Integrated Reconfigurable Photonic Filters based on Interferometric Fractional Hilbert Transforms
}

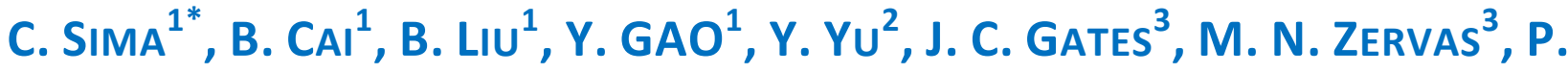 \\ G.R. SMITH ${ }^{3}$, AND D. LIU ${ }^{1}$ \\ ${ }^{1}$ Next Generation Internet Access National Engineering Laboratory, School of Optical and Electronic Information, Huazhong University of \\ Science and Technology, Wuhan 430074, China \\ ${ }^{2}$ Wuhan National Laboratory for Optoelectronics, School of Optical and Electronic Information, Huazhong University of Science and \\ Technology, Wuhan 430074, China. \\ ${ }^{3}$ Optoelectronics Research Centre, University of Southampton, Highfield Campus, SO17 1BJ, Southampton, UK. \\ The first two authors contributed equally to this work \\ *Corresponding author: smct@hust.edu.cn
}

\begin{abstract}
In this paper, we present integrated reconfigurable photonic filters using fractional Hilbert transformers (FrHTs) and optical phase tuning structure within the silica-on-silicon platform. The proposed structure, including grating based FrHTs, an X-coupler and a pair of thermal tuning filaments, is fabricated through the direct UV grating writing technique. The thermal tuning effect is realized by the controllable micro heaters located on the two arms of the X-coupler. We investigate the 200GHz maximum bandwidth photonic FrHTs based on apodized planar Bragg gratings, and analyze the reflection spectrum responses. Through the device integration and thermally modulation, the device could operate as photonic notch filters with $5 \mathrm{GHz}$ linewidth and controllable single sideband (SSB) suppression filters with measured $12 \mathrm{~dB}$ suppression ratio. A $50 \mathrm{GHz}$ instantaneous frequency measuring system using this device is also schematically proposed and analyzed with potential $3 \mathrm{~dB}$ measurement improvement. The device could be configured with these multiple functions according to needs. The reconfigurable structure has great potential in ultrafast all-optical signal processing fields.
\end{abstract}

OCIS codes: Integrated optics devices; Wavelength filtering devices; Waveguides, planar; Gratings

\section{INTRODUCTION}

Integrated reconfigurable photonic processors and filters have been increasingly attractive, such as temporal integration, temporal differentiation and Hilbert transformation [1,2]. The fractional filtering functions are of great interest in information processing, which can improve the characteristics of the processed signal, or be used for additional encoding [3]. For example, the FrHT, which is an advanced Hilbert transform, is of great benefit in optical signal processing. Various photonic FrHTs were demonstrated [3-7], based on asymmetrical apodized FBG operating in reflection [3,4], integrated optical ring resonators [5] and silicon waveguide gratings [6], etc. Some applications of these devices and systems are realized, such as tunable photonic filters, single sideband (SSB) filtering devices and for instantaneous RF signal measurement [1, 8-12]. Some similar integrated schemes with conventional photonic Hilbert transformers (PHTs) were reported [8-10]. In our previous work [8], the conventional PHT and the flattop reflector based on planar Bragg gratings were used to merely obtain the microwave photonic SSB filter. In the literature [9], the identical conventional PHTs are theoretically designed to achieve the nondispersive band-pass filters. In the literature [10], the initial concept of the work was briefly and theoretically introduced. Nevertheless, the filtering functionality and tuning capability is restricted in [8]. Furthermore, both literatures [9, 10] lack of implementation and verification of experimental data while this work comprehensively provides numerical analysis and experimental demonstrations. The device concept and applications in this work are somewhat different compared to references. Furthermore, devices with reconfigurable and programmable features are increasingly important for the photonic integration $[13,14]$.

In this paper, an integrated reconfigurable multifunctional photonic filter within the planar geometry is theoretically proposed and experimentally demonstrated. A symmetric and interferometric planar structure comprising two FrHTs and a couple of micro-heaters is fabricated and characterized. This device allows hundreds of $\mathrm{GHz}$ wideband photonic notch filters, wideband microwave photonic controllable SSB filters and RF signal instantaneous frequency measurement (IFM) according to needs [15]. It is feasible to directly 
configure the filtering response by adjusting the micro heaters and varying the optical field interaction reflected from FrHTs. The optical phase tuning structure also enables the adjustment of the filter performance rapidly and accurately with an enlarged tuning range. Some literatures have experimentally presented FrHTs by the inverse designed method, nevertheless, none of FrHT devices based on directly designed Bragg gratings has been experimentally reported, either in fibers or within integrated platforms [3-6]. In this work, the FrHTs are directly designed and experimentally fabricated with asymmetric grating refractive index apodization profiles, giving a straight and simple way to realize all optical FrHTs. Compared with the literature [8], the incorporated components were entirely modified with the proposed device exhibiting dynamic features and bringing along multiple filtering functionality. The spectrum features and filtering functionalities (e.g. IFM) could not realize with the previously reported PHT [8]. Compared with the literature [9], FrHTs replace the conventional PHTs using asymmetric refractive index apodization profiles, giving different reflection spectra. Additionally, this proposed device has good temperature and mechanical stability since it is monolithically integrated in a single planar chip. Modeled and experimental data are presented to explore the functionalities of the reconfigurable photonic filters.

\section{THEORY AND PRINCIPLES}

The proposed configuration mainly consists of an X-coupler structure, with a photonic FrHT and a micro heater symmetrically located in each arm of the coupler, as shown in Fig. 1. The structure is monolithically integrated in the silica-on-silicon chip with compact geometry. The interaction of the optical signals reflected from the two FrHT gratings occurs at the coupling region. The pair of thermal filaments introduces the optical phase tuning function of the device. Using a pair of thermal filaments instead of single micro-heater could provide larger tuning range and higher tuning rate.

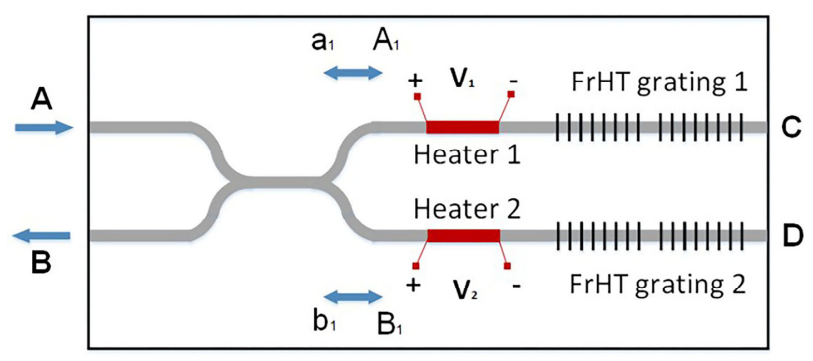

Fig. 1: The scheme of the proposed device.

\section{A. Photonic Fractional Hilbert transform}

Literatures have discussed the definition and features of optical FrHTs [3,5,12]. Generally speaking, FrHTs provide the signal under test a fractional order ' $\rho$ ' of phase shift $\pi$ with a constant amplitude response. In the process of fractional order adjustment, the phase shift can be changed ranging from 0 to $\pi$ controlled by the Bragg grating apodization profile [3]. In the grating apodization profile, $\Delta \mathrm{n}$ is defined as the relative maximum refractive index modulation depths. According to this characteristic, an FrHT can be designed and fabricated directly using a planar Bragg grating. The apodization profile for a physically realizable FrHT is shown in Fig. 2(a), and it could be expressed as below:

$$
\Delta \mathrm{n}(\mathrm{z}) \propto\left\{\begin{array}{cc}
\frac{\sin ^{2}\left[\pi \mathrm{n}_{\mathrm{eff}} \Delta \mathrm{f}\left(\mathrm{z}-\mathrm{z}_{0}\right) / \mathrm{c}\right]}{\mathrm{z}-\mathrm{z}_{0}}, & \mathrm{z}>\mathrm{z}_{0} \\
0, & \mathrm{z}=\mathrm{z}_{0} \\
\varepsilon * \frac{\sin ^{2}\left[\pi \mathrm{n}_{\mathrm{eff}} \Delta \mathrm{f}\left(\mathrm{z}-\mathrm{z}_{0}\right) / \mathrm{c}\right]}{\mathrm{z}-\mathrm{z}_{0}}, & \mathrm{z}<\mathrm{z}_{0}
\end{array}\right.
$$

where $n_{\text {eff }}$ represents the effective refractive index of two gratings, $\left(\mathrm{z}-\mathrm{Z}_{0}\right)$ is the relative position in the apodization function, $\Delta \mathrm{f}$ is the maximum operating bandwidth, calculated at the point in which the spectral magnitude decreases by $3 \mathrm{~dB}$ from the maximum [4], and $\mathrm{c}$ is the light speed. The ratio of refractive index profile at the different side is defined as $\varepsilon$. The relationship between $\varepsilon$ and fractional order $\rho$ was previously analyzed in detail [16].

Assuming that the waveguide structures of the two arms are equivalent for simplicity, when the input optical signal is coupled into port $A$ and propagates through the X-coupler, with general waveguide coupling theory, the optical fields become:

$$
\begin{aligned}
& \mathrm{A}_{1}=\mathrm{A} \sin (\kappa \mathrm{l}) \\
& \mathrm{B}_{1}=-\mathrm{j} A \cos (\kappa \mathrm{l})
\end{aligned}
$$

where $\kappa$ and $\mathrm{l}$ are the coupling coefficient and coupling length respectively. Reflected from FrHT gratings, $\mathrm{A}_{1}$ and $\mathrm{B}_{1}$ are transformed into $\mathrm{a}_{1}$ and $\mathrm{b}_{1}$ respectively. The transformation is expressed as:

(4)

$$
\begin{array}{r}
\mathrm{a}_{1}=\mathrm{A}_{1} \mathrm{Fr}_{1}(\omega) e^{j\left(2 \phi_{1}-\beta L\right)} \\
\mathrm{b}_{1}=\mathrm{B}_{1} \mathrm{Fr}_{2}(\omega) e^{j\left(2 \phi_{2}-\beta L\right)}
\end{array}
$$

where $(2 \phi-\beta L)$ is the total optical phase shift of light, The phase difference caused by thermal tuning between two arms is $\Delta \phi=\beta \Delta \mathrm{L}$ while assuming that the optical path difference between two arms is defined as $\Delta \mathrm{L}$. $\mathrm{Fr}_{1,2}(\omega)$ is the reflection coefficients corresponding to FrHTs. The optical field output from port B is:

$$
\begin{gathered}
B=a_{1}+b_{1}=-j A \sin (\kappa l) \cos (\kappa l) e^{-j \beta \Delta L}\left[F_{1}(\omega) e^{2 j \phi_{1}}+\right. \\
F r_{2}(\omega) e^{2 j \phi_{2}}
\end{gathered}
$$

(5)

\section{B. Thermal tuning}

It has been previously demonstrated that silica-on-silicon planar Bragg gratings fabricated by direct UV writing technique can be thermally tuned [17]. It shows that the phase shift $\Delta \lambda_{B}$ caused by thermal tuning is proportional to Bragg wavelength $\lambda_{B}$ and can be expressed as :

$$
\Delta \lambda_{\mathrm{B}}=\lambda_{\mathrm{B}}(\alpha+\varsigma) \Delta \mathrm{T}
$$

where $\alpha$ is the thermal expansion coefficient corresponding to grating pitch change, and $s$ is the thermo-optic coefficient corresponding to effective index change. Bulk heating experiments show that $\alpha+\varsigma$ is approximately $10 \mathrm{pm} / \mathrm{K}$ in silica-on-silicon substrate in the $1550 \mathrm{~nm}$ wavelength region. This provides a tuning range of $154 \mathrm{GHz}$ and a response speed of $31 \mathrm{GHz} / \mathrm{ms}$ [17]. In this work the thermal tuning of the micro heaters exhibits similar behaviors during the experiments.

\section{SIMULATION AND ANALYSIS}




\section{A. Realization of Fractional Hilbert Transform}

Simulation using the Matlab program was conducted and the results are shown in Fig. 2. Fig. 2(a) shows the apodization profiles for the gratings, Fig. 2(b) and Fig. 2(c) show the amplitude response and phase response of the grating respectively. Here the Bragg gratings are set appropriately, where $\mathrm{L}=20 \mathrm{~mm}$, neff $=1.4478$, and $\Delta \mathrm{f}=150 \mathrm{GHz}$. Since the ratio of refractive index profile at the different side $\varepsilon$ is set to 0.5 in Fig. 2(a), the optical phase shift is $0.73 \pi$ according to the literature [10], thus leading to a fractional order $\rho \approx 0.73$ of phase shift $\pi$ as is shown in Fig. 2(c); here a fractional order PHT (FrHT) is achieved. Plots in blue and red lines also show that the amplitude responses are the same while the phase response are reversed. Furthermore, when changing the ratio $\varepsilon$ (defined as the ratio of refractive index profile at the different side), the fractional order $\rho$ of Hilbert transform would be adjusted as a function of $\varepsilon$, and the relationship is shown in Fig. 2(d) with the subtractive phase shift. The triangle point in Fig. 2(d) denotes the employed FrHT in Fig. 2(a) with blue lines. In all figures showing phase responses, the average group-delay data were compensated for illustration purposes.
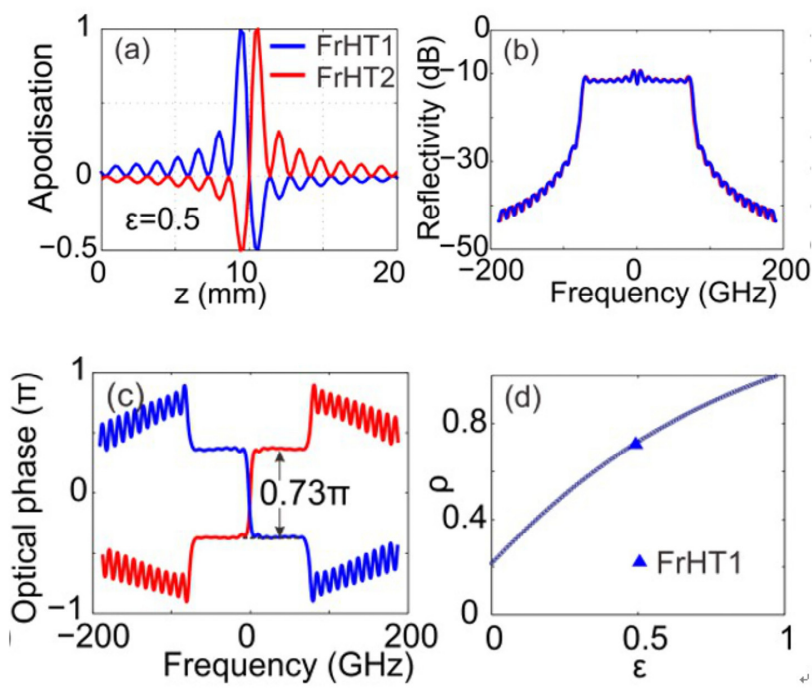

Fig. 2: Simulation: (a) the apodization profiles for two gratings; (b) the amplitude response of two gratings; (c) phase responses of two gratings; (d) the fractional order $\rho$ of Hilbert transform as a function of $\varepsilon$.

\section{B. Different filtering features by thermal tuning}

Using the two different-side-half FrHTs with grating parameters of $10 \mathrm{~mm}$ in length and $200 \mathrm{GHz}$ max bandwidth, we further investigate the phase difference between the two thermal filaments in the proposed interferometric structure. It is found that the amplitude spectrum of this structure varies with the change of optical phase difference $\Delta \Phi$, which is caused by the two micro heaters. Due to the substantial group delay difference of the two gratings located in the same position of each arm, optical signals reflected from the two gratings interact and generate a tilted top of the power spectrum in Fig. 3 , rather diverted from that of classic Hilbert transform. So when there is a fractional order $\rho$ of phase shift $\pi$, e.g. 0.73 , the amplitude response shows a tilted transmission band. Fig. 3(a) indicates the periodic change of the relationship between spectrum and $\Delta \Phi$.

When the optical path difference $\Delta \mathrm{L}$ or $\Delta \Phi$ changes, the performance of the device changes periodically. At first, when $\Delta \Phi=0 \pi$

, the device acts as a photonic notch filter with a maximum bandwidth of about $200 \mathrm{GHz}$. With the increasing $\Delta \Phi$, the flat top starts to be separated and tilted as shown in Fig. 3(b) when $\Delta \Phi$ increases to $0.75 \pi$. In that stage, the device could be used as a photonic SSB filter, and the SSB suppression performance is experimentally presented in the work. Furthermore, it is obvious that when $\Delta \Phi$ reaches $\pi$, the center of the spectrum is enhanced, which is useful for achieving the dual-sideband suppressed filter. At last, when $\Delta \Phi$ increases to $1.25 \pi$, the spectrum become similar but anti-symmetric shape as that with a $0.75 \pi$ phase difference. In applications, we can control and select the side band suppression according to practical needs with the proposed device. For the implementation in microwave photonics, it is also feasible to obtain the wideband notch filter with $\Delta \Phi=0$ or suppressed filter with $\Delta \Phi=\pi$, as well as the selective single-sideband suppression with $\Delta \Phi=0.75 \pi$ or $1.25 \pi$. Through using the micro-thermal elements, the proposed device could achieve different tunable and reconfigurable filtering function.
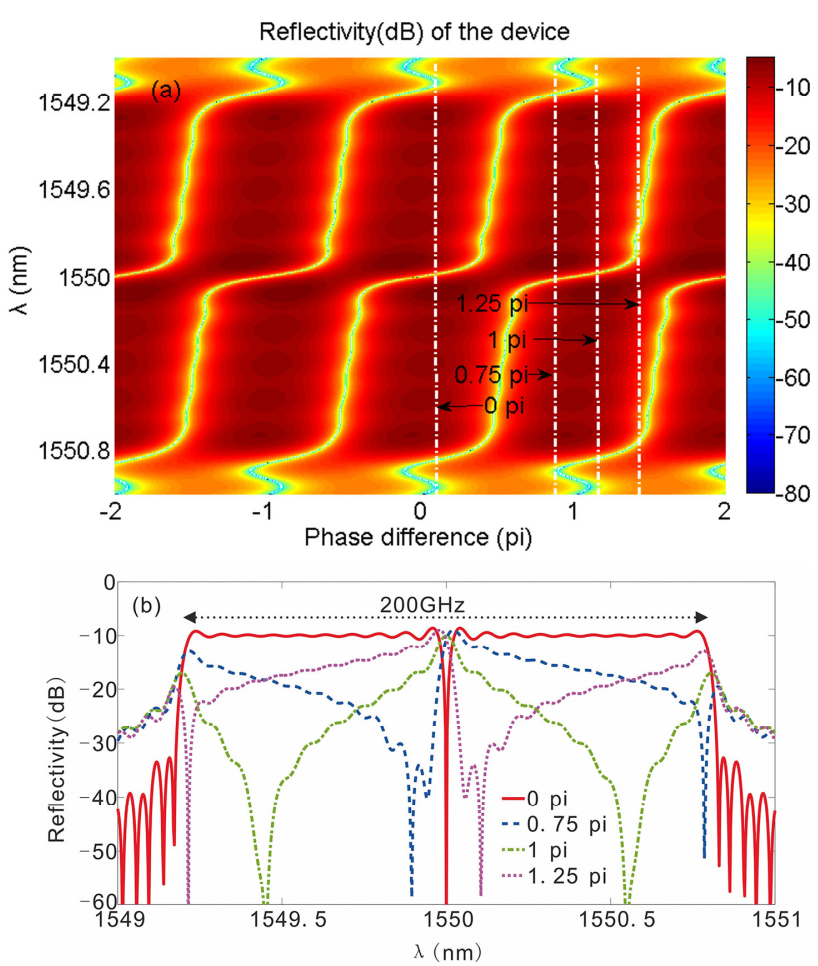

Fig. 3: (a) modelled data of the output spectrum at port $B$, as a function of optical phase difference; (b) four different scenarios if adjusting optical phase difference.

\section{EXPERIMENT AND DISCUSSION}

The integrated devices were fabricated using the direct UV grating writing technique [18], which could provide the fabrication accuracy with microns level and with no need of cleanroom environment. The fabricated chip had $20 \times 10 \times 1 \mathrm{~mm}$ in dimensions and a three-layer silica-on-silicon substrate, showing excellent refractive index uniformity thorough the waveguide. The Ge doped core layer was about $6 \mu \mathrm{m}$ thick, allowing the transmission of a single transverse mode. The monolithically-integrated interferometric structure could reduce the influence caused by the environmental fluctuation due to the cancellation effect of the two arms and then could provide much better stability than some other methods, such as fiber-based structures [11].

The length of the two gratings were set to $10 \mathrm{~mm}$ while the mode neff was measured 1.447 . The coupling region was in about $7.5 \mathrm{~mm}$ 
distance with a $2.5^{\circ}$ crossing angle, and the coupling ratio is about 50 : 50. The arms of X-coupler are fabricated $250 \mu \mathrm{m}$ apart, providing coupling to a standard dual port fiber V-groove assembly. This interferometric structure with a pair of micro-heaters allows larger tuning range and higher tuning rate. The grating parameters are: $\mathrm{L}=$ $10 \mathrm{~mm}, \lambda_{\mathrm{B}}=1549.1 \mathrm{~nm}, \Delta \mathrm{n}=3.6 \times 10^{-4}, \Delta \mathrm{f}=200 \mathrm{GHz}$ and the fractional order $\rho=0.73$, in different sides.
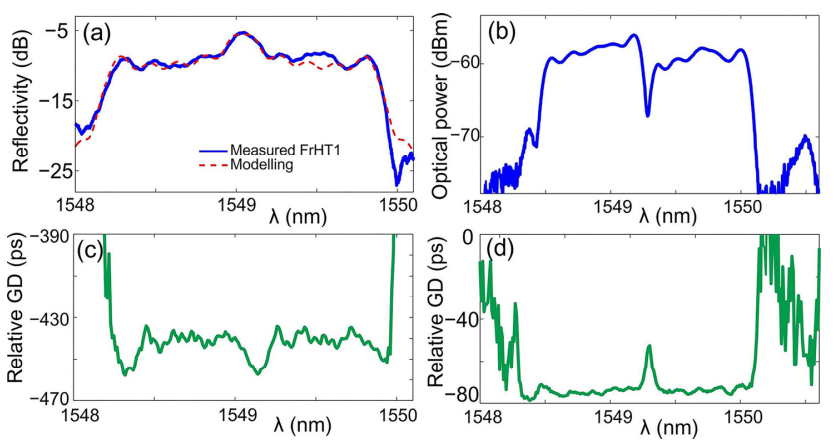

Fig. 4: (a)Amplitude response and (c) relative group delay of FrHT1 from port $\mathrm{C}$ with comparison between measured result (blue solid line) and modelled data (red dashed line); (b) The device output power and (d) relative group delay measured at port B.

Fig. 4(a) shows the amplitude response for the FrHT1 grating. For comparison, the measured data directly obtained from port $\mathrm{C}$ are in blue solid line, while the modelled data are in red dashed line. Fig. 4(b) presents the device output optical power at port B. The output of port $\mathrm{B}$ is the combination of the reflected light from both the two gratings, thus not complementary to port $\mathrm{C}$. Both ports were measured by an OSA together with an erbium doped fiber ASE source and a $3 \mathrm{~dB}$ coupler. Fig. 4(c) and (d) illustrate the measured relative group delay responses, respectively.

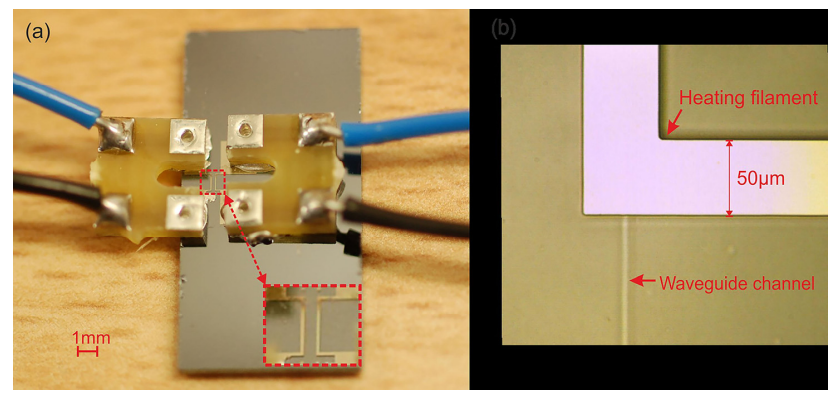

Fig. 5: (a) The camera image of the device with a pair of heating elements; (b) the microscope image of the Nichrome filament $(50 \mu \mathrm{m}$ wide) deposited on top of the waveguide channel.

By using electron beam metal evaporation and photolithography, a pair of $200 \mathrm{~nm}$ thick and $50 \mu \mathrm{m}$ wide Nichrome filaments was deposited in the identical position on top of the two arms of the interferometric waveguide and connected with electric wires, as is shown in Fig. 5. The optical waveguide could be observed in Fig. 5(b). When the Nichrome filaments are applied DC voltages, the optical phase of signals propagating along the waveguide channels would be tuned separately and the optical phase tuning effect is achieved.

Fig. 6 shows a comparison between modeled data and measured results of the device output optical power spectrum, with increased voltages applied to Heater1 in the device. The tilt of the flat part in Fig. 6(a) shows that there was initial phase difference between the two arms, and it was compensated when $3.5 \mathrm{~V}$ was added on Heater1, as Fig. 6(b) shows. This actually allow a photonic notch filter. The measured FWHM of the central notch is $\sim 0.08 \mathrm{~nm}(10 \mathrm{GHz})$ for this device. The central notch could be further narrowed if the apodization profile of the FrHT grating is modified.

Fig. 6(c) and Fig. 6(d) show the different directions of tilt with 9V and $11 \mathrm{~V}$ respectively added on the Heater1. It is obvious that the direction of the tilt of the reflection spectrum changes with the increasing of the phase difference between the arms. To be noted, there is certain divergence between the simulation and measurement in Fig.6, mainly occurs at the optical power level below $-30 \mathrm{dBm}$. This is principally due to the noise floor of the measurement setup. The optical power data in Fig.6 are all measured by an OSA together with an erbium doped fiber ASE source, a $3 \mathrm{~dB}$ coupler, a fiber isolator and an inline polarizer. This characterization setup has the noise floor around $-35 \mathrm{dBm}$ to $-40 \mathrm{dBm}$. Moreover, instead of the entire spectral range, the spectral features near the central wavelength of the measured output power are primarily employed, which has decent agreement with the simulation. The following sections will investigate these filtering functions in detail.
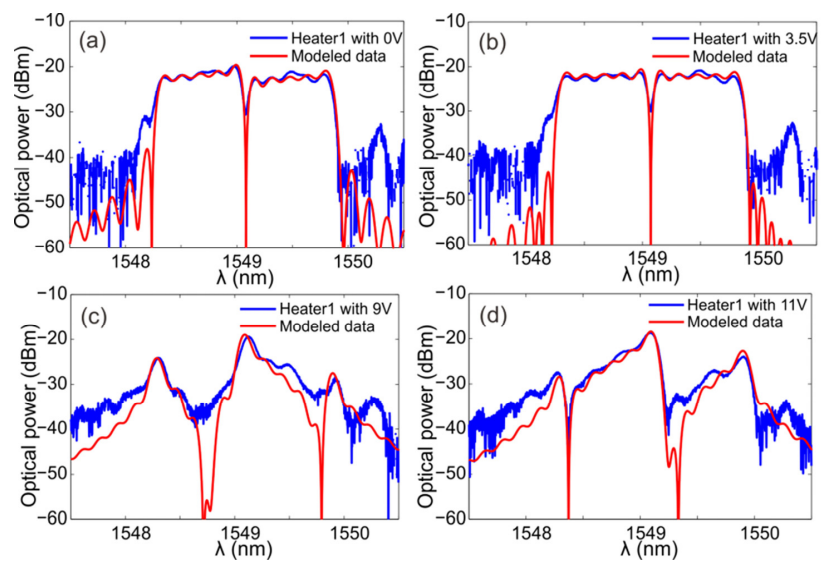

Fig. 6: The modelled data (red line) and measured results (blue line) of the device output power spectrum with Heater1 applied with DC voltages of (a) 0V, (b) 3.5V, (c) $9 \mathrm{~V}$ and (d) $11 \mathrm{~V}$.

\section{A. Wideband photonic notch filters}

Grating based notch filters have applications in multichannel WDM systems and microwave photonic signal processing [19]. As for the photonic notch filters, the proof-of-principle device with the scenario in Fig. 7(a) was employed and analyzed. In the experiment, the grating length is set to $10 \mathrm{~mm}$ long while $n_{\text {eff }}=1.4478$, and $\Delta \mathrm{f}=200 \mathrm{GHz}$. The maximum bandwidth of the photonic filter is about $200 \mathrm{GHz}$ while the FWHM of the central notch is measured to be about 40pm, equivalent to $5 \mathrm{GHz}$. The notch depth of the experimental data is around $10 \mathrm{dBm}$, mainly due to the OSA settings and the rather weak received optical power $(\sim-23 \mathrm{dBm})$ in the experiment. Fig. $7(\mathrm{~b})$ shows the further simulation and investigation for FWHM data of the photonic notch filter. To enhance the typical features of the notch filter, the device is modelled with different truncated grating length of $20 \mathrm{~mm}, 40 \mathrm{~mm}$ and $60 \mathrm{~mm}$ respectively while the $\Delta \mathrm{f}$ remains constant. The results show that the FWHM of the central notch is $10.4 \mathrm{pm}, 15.8 \mathrm{pm}$ and $30.6 \mathrm{pm}$ respectively, with an operating bandwidth of about $200 \mathrm{GHz}$.

Fig. 7(c) shows the relationship between the FWHM of the central notch and the grating length together with operation bandwidth. It is obvious that the FWHM of the center notch remains unaffected when the operating bandwidth $\Delta \mathrm{f}$ increases. Nevertheless, the FWHM data 
has rapid variation when the grating length changes with less than $30 \mathrm{~mm}$ range. If the grating is longer than $40 \mathrm{~mm}$, the change of grating length has minor impact on FWHM data. Although the 3-dB bandwidth FWHM is not as narrow as some other devices, the bandwidth of the device and the nulling depth is desirable; the 3-dB notch filter bandwidth can be changed when the grating length changes, thus getting a suitable 3-dB bandwidth according to real needs. Further simulation results show that it is possible to allow the FWHM of the center notch tuned in $4-40 \mathrm{pm}$, equivalent to $0.5-5 \mathrm{GHz}$ with optimized grating designs.
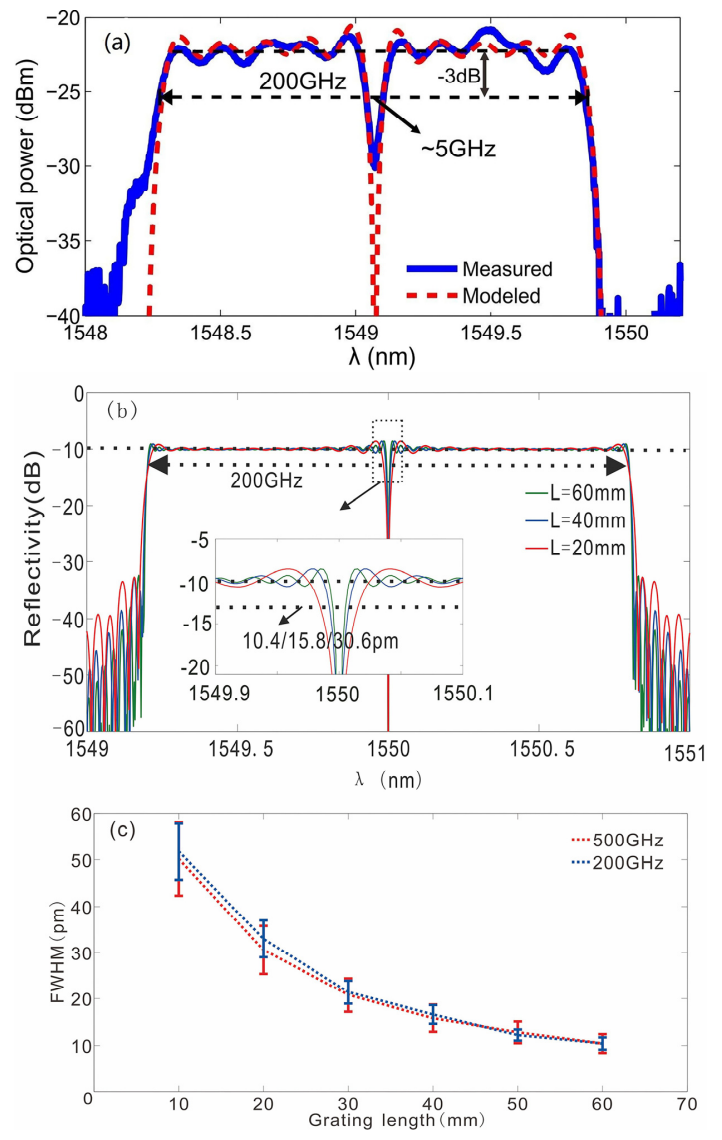

Fig. 7: (a)The measured notch filter with a grating length of 10mm; (b) simulation results with different grating length; (c)simulated FWHM data of the central notch with different grating length and operation bandwidth.

\section{B. Tunable microwave photonic SSB filter}

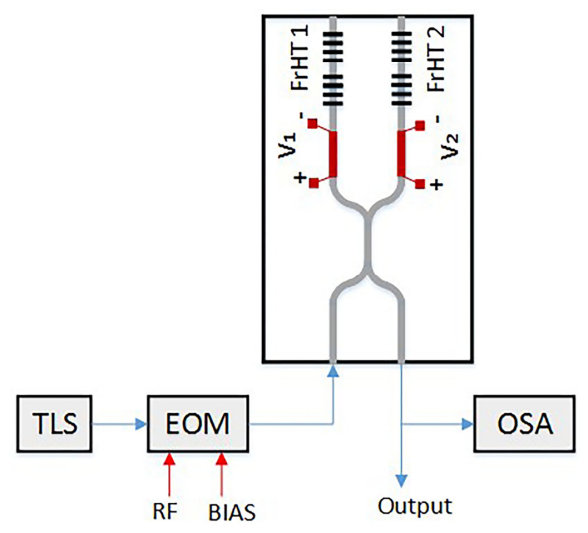

Fig. 8: The RF signal operation setup for the optical SSB filtering system. Enlarged planar chip is for illustration purpose.

As for the tunable microwave photonic SSB filter, the device is used and characterized using the RF signal operation setup in Fig. 8. The optical carrier signal is provided by a tunable laser source (TLS, Agilent $81600 \mathrm{~B}$ ) and it is polarized. The EOM is modulated in amplitude response by the RF signal from a VNA (Agilent8753ES). The output signal of the EOM shows a feature of double sideband (DSB), and is then coupled into port $A$. The device output can be measured directly from port $\mathrm{B}$ by an OSA.
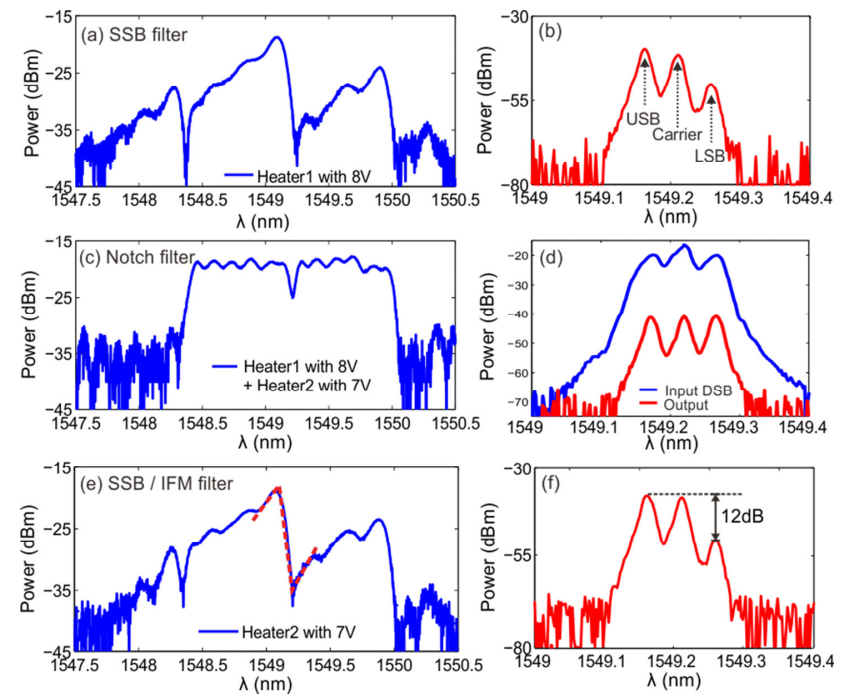

Fig. 9: (a) SSB filter: The measured optical power spectrum with $8 \mathrm{~V}$ applied to Heater1; (b) the LSB suppression with 6GHz RF signals, with the optical spectrum shown in (a); (c) Notch filter: the measured spectrum with $8 \mathrm{~V}$ applied to Heater1 and 7V applied to Heater2; (d) the input 6GHz RF DSB signals (blue line), with the optical carrier frequency suppressed output spectrum; (e) SSB filter: the measured spectrum with 7V applied to Heater2; (f) the 12dB LSB suppression of $6 \mathrm{GHz}$ RF signals, with the optical spectrum shown in (e).

The input DSB signal and all-optical SSB filtering performance are illustrated in Fig. 9. Fig. 9(a) and (b) show the measured optical power spectrum with $8 \mathrm{~V}$ applied to Heater1 and the LSB suppression with $6 \mathrm{GHz}$ RF signals. If Heater2 is also applied with voltages, the spectra will be pulled or pushed to the adjacent state. For SSB modulation of different microwaves, different DC voltages could be applied to the 
micro-heaters to achieve the desired suppression ratio. When Heater2 was applied with $7 \mathrm{~V}$, the optical spectrum altered into a flat top which results in a DSB signal, as Fig. 9(c) and Fig. 9(d) exhibit. The optical spectrum in Fig. 9(c) is similar to that in Fig. 6(b). If the Heater1 thermal tuning was removed but Heater2 remained, the optical spectrum would become that in Fig. 9(e) and the SSB suppression ratio of $\sim 12 \mathrm{~dB}$ in $6 \mathrm{GHz} R$ R frequency was obtained, as is shown in Fig. 9(f). The suppression ratio is closely related to the microwave characterization setup and could be further improved; this could be referred to the discussion in [11]. The microwave signal is generated by the VNA (Agilent8753ES), with the frequency range of $300 \mathrm{KHz}$ to $6 \mathrm{GHz}$. That restricts the highest modulated microwave frequency of 6GHz. The data in Fig.9(a) is the case of specific thermal tuning for the $6 \mathrm{GHz}$ microwave signal, showing the $12 \mathrm{~dB}$ suppression ratio. Microwave signals with bandwidth over $6 \mathrm{GHz}$ were not characterized due to the lack of available microwave generation and detection equipment.

Furthermore, analyzing the spectral data shown in Fig. 9(e), the features (shown as red dashed line in Fig. 9(e)) would be used as the amplitude comparison function (ACF) and provide a route for instantaneous frequency measurement (IFM) in microwave photonics, discussed in the following part.

\section{Instantaneous frequency measurement approach}
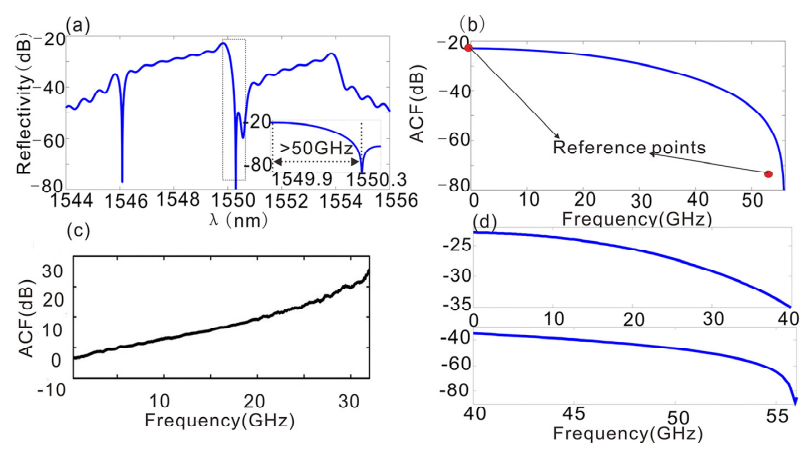

Fig. 10(a), (b) the response spectrum and ACF curve for IMF filter; (c) the reported ACF in literature for comparison [15]; (d) ACF curves in low and high frequency regions respectively.

The reflection spectrum of the device previously shown in Fig. 6(b) and Fig. 6(d) are both feasible to implement the microwave photonic instantaneous frequency measurement (IFM). The essential concept is to investigate and establish the relationship between the unknown microwave signal frequency and the detected output power, which has been thoroughly demonstrated in the literature [15,20]. In the center of the output spectrum, the power is wavelength-dependent, as shown in Fig. 9(e). A monotonic curve is obtained, that can be used as the amplitude comparison function (ACF). From ACF curves, we can confirm the frequency of an unknown RF signal through the optical power measured by the output port.

Considering the current photonic IFM techniques, due to the lack of the corresponding microwave equipment, some modeling work was conducted. In the simulation, the device with $1 \mathrm{THz}$ bandwidth is employed for illustration purpose, and the central area of the spectrum is enlarged and shown in Fig. 10(a). The left right side interception part in the central area could be used as the ACF, as shown in Fig. 10(b). Fig. 10(b) shows that this device can be used in IFM system with an ultra-wide bandwidth up to 50GHz. Fig. 10(c) shows the ACF data curve in the literature for comparison [15]. The ACF shown in Fig.
10 (b) has an increased slope rate of $\sim 1.1 \mathrm{~dB} / \mathrm{GHz}$ in the whole range, compared to $\sim 0.9 \mathrm{~dB} / \mathrm{GHz}$ in Fig. 10 (c). It is concluded that if the identical experimental set-up is employed, the proposed device in this work could reduce the measurement error to $618 \mathrm{MHz}$, compared to the error of $755 \mathrm{MHz}$ in the literature [15]. Furthermore, Fig. 10(d) shows the ACF curves in $0-40 \mathrm{GHz}$ and $40 \mathrm{GHz}-50 \mathrm{GHz}$ frequency regions respectively. In the frequency range of $40-50 \mathrm{GHz}$, the system exhibits an advanced measurement resolution with a slope rate of $\sim 2 \mathrm{~dB} / \mathrm{GHz}$ in the higher frequency domain, where the measurement error is estimated about $340 \mathrm{MHz}$. Hence, the device would allow a potential $3 \mathrm{~dB}$ improvement in the measurement resolution and measurement error in the $40 \mathrm{GHz}-50 \mathrm{GHz}$ frequency region.

Fig. 11 shows the proposed system schematic of the IFM approach using the fabricated photonic filter device. Modulated by frequency-unknown RF signals, the optical carrier is sent to the device, and the output power is measured by a photodiode (PD). Once the PD detects the output power of the unknown signal, the signal frequency can be obtained according to the ACF. In the practical applications, the output power magnitude of the IFM device cloud be effected while the shape of the ACF curve remains the same. It is proposed that two or more reference points could be firstly introduced to determine the ACF magnitude by measuring the signals whose frequency are already known. To be noted, the device has the total insertion loss of around $20 \mathrm{~dB}$ and the optical amplifier (e.g. EDFA) is required in practical applications.

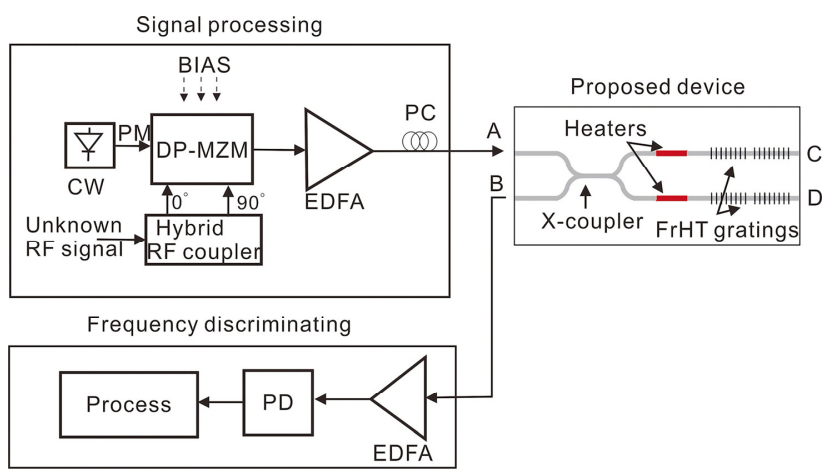

Fig. 11: The proposed schematic for the RF signal instantaneous frequency measurement (IFM) system using the fabricated device.

\section{CONCLUSION}

We propose and experimentally demonstrate tunable and reconfigurable optical signal filters based on the integrated photonic interferometric fractional order Hilbert Transformers. The compact and integrated photonic device was fabricated through direct UV grating writing technique while the reconfigurable photonic filter was realized by the interferometric structure. The device could be employed as wavelength tunable wideband notch filters, controllable SSB filters as well as for instantaneous frequency measurement. The theoretically simulation and experimental data of the proposed photonic filters are comprehensively presented and discussed. This integrated and reconfigurable device shows great potentials in ultrafast all-optical signal processing.

Funding Information. National Natural Science Foundation of China (NSFC 61404056, 61475050, 61290315). Natural Science Foundation of Hubei Province (2017CFB258).

Acknowledgment. The authors would like to thank Prof. Jianji Dong and Dr. Li Shen at the Wuhan National Laboratory for Optoelectronics (WNLO) for the device modeling discussion. 


\section{References}

1. W. Liu, M. Li, R. S. Guzzon, E. J. Norberg, J. S. Parker, M. Lu, L. A. Coldren, and J. Yao, "A fully reconfigurable photonic integrated signal processor," Nat. Photon. 10, 190-195 (2016).

2. M. Li, Sun. S, Malacarne. A, Larochelle. S, Yao. J, Zhu. N and Jose. N, "Reconfigurable single-shot incoherent optical signal processing system for chirped microwave signal compression." Sci. Bull. 62, 242-248 (2017).

3. C. Cuadrado-Laborde, "Proposal and design of a photonic in-fiber fractional Hilbert transformer," Photon. Technol. Lett. 22, 33-35, (2010).

4. M. Li and J. Yao, "Experimental demonstration of a wideband photonic temporal Hilbert transformer based on a single fiber Bragg grating," Photon. Technol. Lett. 22, 1559-1561 (2010).

5. L. Zhuang, M. R. Khan, W. Beeker, A. Leinse, R. Heideman, and C. Roeloffzen, "Novel microwave photonic fractional Hilbert transformer using a ring resonator based optical all-pass filter," Opt. Express. 20, 26499-26510 (2012).

6. M. Burla, M. Li, L. R, X. Wang, M. R. F-R, L. C, and J. A, "Terahertz-bandwidth photonic fractional Hilbert transformer based on a phase-shifted waveguide Bragg grating on silicon." Opt. Lett. 39, 6241-6244 (2014).

7. B. Liu, C. Sima, W. Yang, B. Cai, D. Liu, Y. Yu, J Gates, M. Zervas, P. Smith. "Experimental Investigation of Large Time-Bandwidth Product Photonic Hilbert Transformer based on compact Bragg grating," IEEE PHOTONICS J. 8, 1-8 (2016).

8. C. Sima, J. C. Gates, H. L. Rogers, et al. "Phase controlled integrated interferometric single-sideband filter based on planar Bragg gratings implementing photonic Hilbert transform," Opt. Lett. 38, 727-729 (2013).

9. H. P. Bazargani, M. R. Fernándezruiz, and J. Azaña. "Tunable, nondispersive optical filter using photonic Hilbert transformation," Opt. Lett. 39, 5232-5235 (2014).
10. B. Cai, C. Sima, B. Liu, D. Liu, Y. Yu, J. Gates, and P. Smith, in Asia Communications and Photonics Conference 2016 (Optical Society of America, 2016), p. AS3F.4.

11.Z. Li, H. Chi, X. Zhang, and J. Yao, "Optical Single-Sideband Modulation Using a Fiber-Bragg-Grating-Based Optical Hilbert Transformer," Photon. Technol. Lett. 23, 558-560 (2011).

12.M. Li, and J. Yao, "All-fiber temporal photonic fractional Hilbert transformer based on a directly designed fiber Bragg grating," Opt. Lett. 35, 223-225 (2010).

13. N Photonics, "Birth of programmable optical chips," Nat. Photon. 10, 1038-1039 (2016).

14. Lowery, A. J., Roeloffzen, C. G. H., Boller, K., Zhuang, L., and Hoekman, M, "Programmable photonic signal processor chip for radiofrequency applications," Optica. 2, 854-859 (2015).

15. Burla, M. Wang, X. Li, M. Chrostowski. L, and Azaña. J "Wideband dynamic microwave frequency identification system using a low-power ultracompact silicon photonic chip," Nat. Comm. 7, 13004-13012 (2016).

16. B. Liu, C. Sima, W. Yang, D. Liu, Y. Yu, J. C. Gates, M. N. Zervas, and P. G.R. Smith, in Conference on Lasers and Electro-Optics Pacific Rim 2015 (Optical Society of America, 2015), p. 27P_78.

17. C. Holmes, D. O. Kundys, J. C. Gates, C. B. E. Gawith, and P. G. R. Smith. "150 GHz of thermo-optic tuning in direct uv written silica-on-silicon planar Bragg grating," ELECTRON LETT. 45, 954-956 (2009).

18. C. Sima, J. C. Gates, H. L. Rogers, P. Mennea, C. Holmes, M. N. Zervas, and P. G.R. Smith. "Ultra-wide detuning planar Bragg grating fabrication technique based on direct UV grating writing with electro-optic phase modulation," Opt. Express. 13, 15747-15754 (2013).

19.X. Zou, M. Li, W. Pan, L. Yan and L. Shao "Multichannel Narrow, Flat-Top Optical Filters Based on Multiple-Phase-Shifted and Phase Sampled FBG," IEEE J Quantum Elect. 53, 1-5, (2017).

20.J. Hervás, A. L. Ricchiuti, W. Li, N. Zhu, C. R.F-Pousa, S. Sales, M. Li and J, $\mathrm{Ca}$ [many, "Microwave Photonics for Optical Sensors," in IEEE J. Sel. Top. Quant. 23, 327-339,

(2017). 